\title{
De novo and comparative transcriptomic analysis explain morphological differences in Panax notoginseng taproots
}

\section{Lifang Yang}

Kunming University of Science and Technology

Hanye Wang

Kunming University of Science and Technology

\section{Panpan Wang}

Kunming University of Science and Technology

\section{Mingju Gao}

Wenshan University

\section{Luqi Huang}

National Resource Center for Chinese Materia Medica, Chinese Academy of Chinese Medical Sciences

\section{Xiuming Cui}

Kunming University of Science and Technology

Yuan Liu ( $\sim$ liuyuan513@kust.edu.cn )

Kunming University of Science and Technology

\section{Research Article}

Keywords: Panax notoginseng, Taproot, Phenotypic differences, De novo assembly transcriptome, Differentially expressed genes

Posted Date: October 21st, 2021

DOl: https://doi.org/10.21203/rs.3.rs-963388/v1

License: (c) (1) This work is licensed under a Creative Commons Attribution 4.0 International License. Read Full License

Version of Record: A version of this preprint was published at BMC Genomics on January 31st, 2022. See the published version at https://doi.org/10.1186/s12864-021-08283-w. 


\section{Abstract}

Background: Panax notoginseng (Burk.) F. H. Chen (PN) belonging to the genus Panax of family Araliaceae is widely used in traditional Chinese medicine to treat various diseases. PN taproot, as the most vital organ for the accumulation of bioactive components, presents a variable morphology (oval or long), even within the same environment. However, no related studies have yet explained the molecular mechanism of phenotypic differences. To investigate the cause of differences in the taproot phenotype, de novo and comparative transcriptomic analysis on $\mathrm{PN}$ taproot was performed.

Results: A total of $133,730,886$ and $114,761,595$ paired-end clean reads were obtained based on highthroughput sequencing from oval and long taproot samples, respectively. 121,955 unigenes with contig $\mathrm{N} 50=1,774 \mathrm{bp}$ were generated by using the de novo assembly transcriptome. The annotation results showed 38,313 BLASTX hits and 24,820 BLASTP hits. KEGG analysis identified 52 genes encoding class III peroxidase (PRX) family members, 13 genes encoding L-Ascorbate peroxidase (APX) family members, and 99 genes encoding a series of mitogen-activated protein kinase (MAPK) family members.

Differentially expressed genes analysis indicated substantial upregulation of genes encoding APX3 and PRX45 enzymes, which are related to reactive oxygen species metabolism, and the MPK3 enzyme, which is related to cell proliferation and plant root development, in long taproots compared with that in oval taproots. Furthermore, the determination results of real-time quantitative PCR, enzyme activity, and $\mathrm{H}_{2} \mathrm{O}_{2}$ content verified transcriptomic analysis results.

Conclusion: These results collectively demonstrate that reactive oxygen species (ROS) metabolism and the MPK3 enzyme may play vital roles in regulating the taproot phenotype of PN. This study provides further insights into the genetic mechanisms of phenotypic differences in other species of the genus Panax.

\section{Introduction}

Panax notoginseng (Burk.) F. H. Chen (PN) is a perennial herb belonging to the Araliaceae family. PN taproot, as the most vital organ for the accumulation of bioactive components, is a raw material of many famous patented Chinese medicines, including Yunnan Baiyao, Xuesaitong, and Compound Danshen Dripping Pills [1, 2]. The main bioactive components isolated from PN taproots include ginseng saponins, notoginsenoside, and dencichine [2,3]. Extensive pharmacology studies have shown that PN prevents cardio- and cerebrovascular disease, has anti-inflammatory effects, and aids immune regulation [4, 5]. Xuesaitong, produced from the total saponins extracted from PN taproots, is in broad clinical use for the prevention and treatment of hyperlipidemia, coronary heart disease, stroke sequelae, and other chronic diseases, and is particularly favored by patients. In general, the growth rate of PN is relatively slow in the wild, and even with good field management it takes at least three years to grow and accumulate sufficient bioactive components in its taproots before they can be harvested and used as a medicinal material. However, in the process of PN cultivation, there has been a perplexing phenomenon wherein there are obvious differences in the root phenotype during growth, even when many PN plants are grown in the 
same environment. Specifically, some of them present long strip-like ginseng (long taproot of PN: LPN), whereas others are oval-like (oval taproot of PN: OPN); they are called "Luobo qi" or "Chang qi" and "Geda qi" or "Tuan qi," respectively.

To date, many studies have focused on the biosynthetic pathways, whole-genome expression profile, and pharmacological effects of the active ingredients from PN taproots [6-12]. To the best of our knowledge, no studies have yet investigated the cause of morphological differences in PN taproots, and the metabolic regulation pathways involved in taproot morphology differences remain unknown. Plant traits are shaped by two vital factors, external environment condition and genetic regulation. To a large extent, visible changes in plants are caused by a series of invisible physiological changes and molecular regulation based on transcriptional expression levels, which reveal that gene expression changes are closely correlated with wide variations in plant development characteristics [13]. In recent years, many researchers have sought to understand the regulation mechanism of plant root anatomy and architecture by using the model plant Arabidopsis thaliana and the diverse metabolic pathways involved in root development regulation [14]. Consistent with the growth of plant aerial parts, the formation and development of roots follow the internal laws of gene regulation [15]. In other words, changes in root morphology are inevitably accompanied by changes in the internal metabolic pathways. Therefore, research based on transcriptional regulation reveals the causes of plant morphological changes from the perspective of molecular mechanisms. Moreover, the rapid advances in next-generation sequencing (NGS) can provide abundant transcriptome data, which offers insights into the molecular regulation mechanism of plant growth and development, such as rhizome formation in Nelumbo nucifera, taproot thickening in PN, fruit morphology of pumpkin cultivars, secondary metabolite accumulation in tuberous roots of Aconitum heterophyllum, and improvement of disease resistance in strawberries [16-20]. Collectively, analysis of these data can reveal the internal molecular interaction mechanisms of various plant types, including morphology, yield, and disease resistance.

Both reactive oxygen species (ROS) and class III peroxidase (PRX) play pivotal roles in modulating plant root development. Either ROS balance or PRX activity affects plant root growth and elongation, and this pathway is completely independent of the signaling pathways of plant hormones, such as cytokinin and auxin; this has long been confirmed in A. thaliana [21-23]. Additionally, numerous studies have confirmed that ROS are important plant growth regulators and widely involved in various processes of plant root development, such as meristematic expansion and root elongation [24-31]. ROS homeostasis maintains a delicate balance under the coordination of ROS production and scavenging, maintaining an appropriate threshold boundary between redox potential and cytotoxicity [32]. Two important enzymatic families, PRX and NADPH oxidase family, contribute to ROS production [26,33]. ROS scavenging is a two-armed regulation mechanism. One arm comprises antioxidative enzymes, including catalase (CAT), L-Ascorbate peroxidase (APX), PRX, superoxide dismutase (SOD), and glutathione peroxidase (GPX) [34-37]. The other arm consists of nonenzymatic antioxidants, such as reduced glutathione, ascorbic acid, and flavonoids [38]. It has already been reported that oxidative stress in plant cells is regulated by the mitogen-activated protein kinase (MAPK) family cascade [39]. The MAPK family widely participates in biological processes 
in plants, such as cell proliferation and differentiation, as well as responding to and tolerating diverse stresses and environmental stimuli [39-41].

Therefore, to investigate the causes of phenotypic differences between LPN and OPN at the molecular regulation level, we performed an in-depth study of the transcriptome data of PN taproot and then verified the results. Three genes were very likely involved in regulating the taproot morphogenesis of PN, which provides a reliable explanation for the phenotypic variation of PN taproot.

\section{Materials And Methods}

\section{Plant materials}

PN (three years old) was collected at the vigorous growth stage from three sampling sites: SP

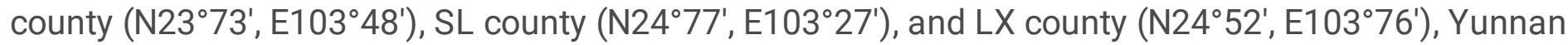
Province, People's Republic of China. The specimens undertook the formal identification by Xiuming Cui that have been preserved in the greenhouse of the Faculty of Life Science and Technology, Kunming University of Science and Technology. Taproots with obviously different phenotypes (LPN and OPN) were collected separately from individual plants and washed, followed by measurement of their length and width. The samples were immediately stored in liquid nitrogen for further processing. Ten samples were collected from each sampling site, including five LPN and five OPN samples; thus, a total of 30 samples were collected from three sampling sites.

\section{RNA isolation, library construction, and sequencing}

Five samples each of LPN and OPN were collected from each sampling site, for a total of three mixed samples each of LPN and OPN (marked as LPN1, LPN2, and LPN3 for LPN and as OPN1, OPN2, and OPN3 for OPN) for RNA extraction and further experimentation. Total RNA per mixed sample was extracted from the taproot tissue using the RNeasy Plus Mini kit (Qiagen, Valencia, CA, USA), and purified using oligo (dT) magnetic beads. After completing both cDNA library synthesis and PCR amplification for each mixed sample, the PCR product was purified on an AMPure XP system (Beckman Coulter, Beverly, MA, USA). RNA integrity was assessed using an Agilent 2100 Bioanalyzer Instrument (Agilent Technologies, Santa Clara, CA, USA), and RNA degradation and contamination were monitored on $1 \%$ agarose gels.

One cDNA library was constructed from each mixed sample, and a total of six libraries were generated. Clustering of the index-coded samples was performed using TruSeq PE Cluster Kit v3-cBot-HS (Illumina, San Diego, CA, USA) based on the cBot Cluster Generation System, following the manufacturer's instructions. After cluster generation, 150 bp paired-end sequencing was performed using NGS based on the HiSeq 2500 system (Illumina). The reads were then sequenced and cleaned to generate clean data by removing adapter, ploy- $\mathrm{N}$, and low-quality reads.

\section{Quality control and de novo assembly}


The quality of clean data was evaluated using FASTQC software v0.11.9 (https://www.bioinformatics.babraham.ac.uk/projects/fastqc/). Sequenced reads with a per base average quality score below 28 were filtered, and the first 15 bases of each read were removed using Trimmomatic software v0.39 [42]. To create a reference transcriptome of PN taproot for further analysis, we combined all trimmed reads into one sample using Trinity v2.8.4 [43] to complete the assembly process for a transcriptome. Then, the assembled transcriptome was clustered, and redundancy was removed using CD-HIT-EST software v4.8.1 [44] with a similarity parameter of 0.95. The longest transcripts were extracted as a reference transcriptome (namely unigenes) for subsequent functional annotation and DEG analysis between LPN and OPN.

\section{Functional annotation and analysis}

Trinotate pipeline v3.2.0 [43] was used to annotate both the reference transcriptome and protein-coding regions predicted by TransDecoder v5.5.0. Trinotate pipeline can integrate several databases including SwissProt, RNAmmer v1.2 (predicting ribosomal RNA), SignalP v5.0 (predicting signal peptides), TMHMM v2.0 (predicting transmembrane helices), and HMMER v3.3.1 (identifying protein domains) to populate its own database. In addition to the databases mentioned above, Trinotate can map BLAST results of the transcripts to $\mathrm{GO}$ and $\mathrm{KO}$ databases to obtain corresponding $\mathrm{GO}$ and $\mathrm{KO}$ numbers, respectively [45-47]. In brief, Trinotate merged SwissProt, Pfam, and other related databases into the SQLite database. BLASTX

with an E-value cut-off of $1.0 \times 10^{-5}$, BLASTP, RNAmmer, SignalP, TMHMM, HMMER, eggNOG, KEGG, and GO homology searches were performed against the SQLite database. Finally, the search results were compiled in a report file as a table. The subcategories of GO terms of the annotated unigenes were visualized using Panther GO-slim, and the related metabolic pathways found from KEGG annotation were visualized using KEGG Mapper [46, 48].

\section{DEG analysis}

The gene expression level of each sample from LPN and OPN was calculated by mapping clean reads back to the reference transcriptome using RNA-seq by expectation-maximization (RSEM) software v1.3.3 [49] and Bowtie 2 v2.4.1 alignment [50]. The expression matrix generated by RSEM was imported into DESeq2 for DEG analysis between LPN and OPN [51]. Genes with adjusted $p<0.05$ and absolute $\log _{2}$ fold change $>1$ were selected as significant DEGs. Up- and downregulated DEGs in LPN were further investigated by GO enrichment analysis using the Goseq R Bioconductor package and then mapped to KEGG pathways using the Pathview R Bioconductor package [52]. The PPI network diagram based on DEGs was constructed using the STRING database (https://string-db.org) with default parameters, and the PPI network was further optimized using Cytoscape software v3.8.2 [53].

\section{Measurement of $\mathrm{H}_{2} \mathrm{O}_{2}$ content and enzyme activities}

The $\mathrm{H}_{2} \mathrm{O}_{2}$ content in OPN and LPN was determined using the $\mathrm{H}_{2} \mathrm{O}_{2}$ content assay kit (Solarbio, Beijing, People's Republic of China) following the manufacturer's instructions. The outcome was expressed as 
$\mathrm{H}_{2} \mathrm{O}_{2}$ content per gram of fresh taproot weight ( $\mu \mathrm{mol} / \mathrm{g}$ fresh weight). The enzyme activities of APX and PRX in OPN and LPN were measured using APX and PRX assay kits (Solarbio) following the manufacturer's instructions. The enzyme activity was calculated in terms of enzyme activity units per $\mathrm{g}$ of fresh taproot weight ( $\mathrm{U} / \mathrm{g}$ fresh weight). Three biological replicates were used for each experiment.

\section{RT-qPCR validation of unigenes}

Total RNA was extracted from six PN taproot samples using the TRIzol Reagent (Thermo Fisher Scientific, Waltham, MA, USA). cDNA was reverse-transcribed using the RT6 cDNA Synthesis Kit v2 (Beijing Qingke Biotechnology, Beijing, People's Republic of China). Specific primers for each gene were designed for RTqPCR amplification using Primer-BLAST (https://www.ncbi.nlm.nih.gov/tools/primer-blast/). Actin was used as an internal reference gene to normalize the mRNA expression levels of target genes in each sample [54]. The specific primers for the target genes and actin are listed in Supplementary Table 2. Quantitative reactions were performed using the RT-qPCR Detection System (FQD-96A, Hangzhou Bioer Technology, Hangzhou, People's Republic of China). The reaction mixture $(20 \mu \mathrm{L})$ contained $10 \mu \mathrm{L} 2 \times \mathrm{T} 5$ Fast qPCR Mix (SYBR Green I), $0.8 \mu \mathrm{L}$ each of the forward and reverse primers, and $1 \mu \mathrm{L}$ of template cDNA. Finally, qPCR amplification was conducted under the following conditions: $95^{\circ} \mathrm{C}$ for $60 \mathrm{~s}$, followed by 40 cycles of $95^{\circ} \mathrm{C}$ for $15 \mathrm{~s}, 60^{\circ} \mathrm{C}$ for $15 \mathrm{~s}$, and $72{ }^{\circ} \mathrm{C}$ for $30 \mathrm{~s}$. The relative expression of internal reference and target genes was calculated using the $2^{-\triangle \Delta C T}$ method. Three biological replicates were used for the validation study.

\section{Results}

\section{Statistics of phenotype data}

The typical phenotypes of OPN and LPN are shown in Fig. 1a. Length-width ratios of $30 \mathrm{PN}$ taproot samples (OPN and LPN) collected from Shipin (SP), Shilin (SL), and Luxi (LX) county were calculated. Length-width ratios for OPN and LPN samples collected from the SP county were $1.42 \pm 0.23$ and $2.96 \pm$ 0.90 , those from SL county were $1.47 \pm 0.26$ and $2.37 \pm 0.45$, and those from LX county were $1.27 \pm 0.20$ and $1.95 \pm 0.38$, respectively. From the calculated results, the length-width ratios of OPN samples were approximately 1.00 to 1.50 , whereas those of the LPN samples were approximately 2.00 to 3.00 ; thus, the length-width ratio of LPN was approximately twice that of OPN. These data were statistically analyzed using the PASW software v18.0.0. Statistical results (Fig. 1b) indicated that there was a significant difference in the length-width ratio between OPN and LPN taproots for each sampling site $(p<0.05)$.

\section{Illumina sequencing results}

After removing adapter, ploy-N, and low-quality reads, Illumina sequencing produced a total of $133,730,886(39.11 \mathrm{~Gb})$ and 114,761,595 (33.31 Gb) paired-end clean reads from three samples each of OPN and LPN, respectively (Table 1 ). After assessing read quality using FASTQC software and trimming the first 15 bases of each read using Trimmomatic software, the length of the retained reads was $135 \mathrm{bp}$, and all of them had a high-quality score (> 28) for subsequent de novo assembly. 
Table 1 Summary statistics of sequencing data from OPN and LPN using the Illumina HiSeq 2500 system.

\begin{tabular}{|c|c|c|c|c|c|}
\hline $\begin{array}{l}\text { Sample } \\
\text { number }\end{array}$ & $\begin{array}{l}\text { Sample } \\
\text { location }\end{array}$ & $\begin{array}{l}\text { Number of total } \\
\text { reads }\end{array}$ & $\begin{array}{l}\text { Clean bases } \\
\text { (Gb) }\end{array}$ & Q20 (\%) & Q30 (\%) \\
\hline OPN1 & $\begin{array}{l}\mathrm{SP}\left(\mathrm{N} 23^{\circ} 73^{\prime}\right. \\
\left.\mathrm{E} 103^{\circ} 48^{\prime}\right)\end{array}$ & $85,408,766$ & $12.52 \mathrm{~Gb}$ & 97.90 & 93.91 \\
\hline OPN2 & 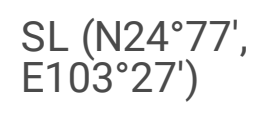 & $86,151,720$ & $12.54 \mathrm{~Gb}$ & 98.52 & 95.40 \\
\hline OPN3 & $\begin{array}{l}\operatorname{LX}\left(\mathrm{N} 24^{\circ} 52^{\prime}\right. \\
\left.\mathrm{E} 103^{\circ} 76^{\prime}\right)\end{array}$ & $95,901,286$ & $14.05 \mathrm{~Gb}$ & 98.00 & 94.17 \\
\hline LPN1 & $\begin{array}{l}\mathrm{SP}\left(\mathrm{N} 23^{\circ} 73^{\prime},\right. \\
\left.\mathrm{E} 103^{\circ} 48^{\prime}\right)\end{array}$ & $72,558,374$ & $10.64 \mathrm{~Gb}$ & 98.09 & 94.32 \\
\hline LPN2 & $\begin{array}{l}\text { SL }\left(\mathrm{N} 24^{\circ} 77^{\prime}\right. \\
\left.\mathrm{E} 103^{\circ} 27^{\prime}\right)\end{array}$ & $78,429,508$ & $11.49 \mathrm{~Gb}$ & 97.47 & 92.88 \\
\hline LPN3 & $\begin{array}{l}\operatorname{LX}\left(\mathrm{N} 24^{\circ} 52^{\prime}\right. \\
\left.\mathrm{E} 103^{\circ} 76^{\prime}\right)\end{array}$ & $78,535,308$ & $11.18 \mathrm{~Gb}$ & 97.61 & 93.26 \\
\hline
\end{tabular}

Abbreviations: OPN, oval taproot of Panax notoginseng (Burk.) F. H. Chen; LPN, long taproot of Panax notoginseng (Burk.) F. H. Chen; SP, Shipin county; SL, Shilin county; LX, Luxi county.

\section{De novo transcriptome assembly and function annotation}

The reference transcriptome consisted of 121,955 unigenes with an average length of $914 \mathrm{bp}$, contig N50 $=1,774 \mathrm{bp}$, and GC content percentage $=38.27 \%$; the length distribution statistics of all transcripts are shown in Fig. 2a. In total, 42,207 protein-coding regions were predicted from the reference transcriptome using the TransDecoder software. The final annotation results showed 38,313 Basic Local Alignment Search Tool (BLASTX) hits and 24,820 BLASTP hits. Moreover, 25,706, 31,269, and 26,563 unigenes were mapped to the Kyoto Encyclopedia of Genes and Genomes (KEGG), Gene Ontology (GO), and eggNOG databases, respectively, and 15,797 unigenes were common among the KEGG, GO, Nr, and eggNOG databases (Fig. 2b).

GO function classification distinguished the annotated unigenes involved in different cellular components, biological processes, and molecular functions, and then divided them into subcategories within each GO term domain. Within the cellular component subcategory (Fig. 3a), the majority of annotated unigenes were in the membrane (31.03\%), organelle $(24.99 \%)$, and nucleus $(16.71 \%)$ subcategories, and others were distributed in the cytoplasm (12.24\%), supramolecular complex (6.75\%), extracellular region (2.25\%), cell wall (1.41\%), and peroxisome (0.64\%) subcategories. Within the biological process subcategories (Fig. 3b), a high percentage of annotated unigenes were involved in metabolic processes $(16.31 \%)$, biological regulation (14.26\%), response to stimulus (13.61\%), and signal transduction and transport (11.2\%). The rest were mainly involved in RNA processing (8.03\%), DNA processing $(9.87 \%)$, protein processing $(8.79 \%)$, development process $(5.60 \%)$, and cell cycle $(3.43 \%)$. 
Within the molecular function subcategories (Fig. 3c), 49.01\% of annotated unigenes were involved in binding, $42.76 \%$ were involved in enzyme activity, $3.45 \%$ were involved in transporter activity, $1.51 \%$ participated in structural molecule activity, $0.58 \%$ participated in calcium channel activity, and $0.52 \%$ participated in translation regulator activity.

\section{Mapping of KEGG pathways}

The analysis of metabolic pathway assignment from the annotated unigenes was performed using the KEGG database. The KEGG annotation results showed that 16,225 unigenes were assigned to specific metabolic pathways with corresponding KEGG Orthology (KO) numbers; among them, 10,575 unigenes matched $\mathrm{KO}$ numbers in the KEGG database for $\mathrm{A}$. thaliana and are involved in various metabolic pathways. Then, the repeated KO numbers were added up and counted. The top $15 \mathrm{KO}$ numbers (with corresponding definition) mapped to enzymes are shown in Fig. 3d. The total number of genes encoding PRX (K00430) ranked fourth, which indicates that PRX may play an extremely important role in the PN taproot development processes. Moreover, all known enzymes involved in the metabolism pathway of the ROS and MAPK families were identified in the KEGG database for $A$. thaliana. Among the genes encoding enzymes related to ROS metabolism, 52 genes encoding PRX family members, 16 encoding GPx family members, and 13 encoding APX family members were identified. In addition, KEGG analysis identified 99 genes that encode a series of MAPK family members related to plant growth, development, and response to oxidative stress, of which 73 were mapped to the KEGG database for A. thaliana, and 21 of the 73 genes were assigned to specific metabolic pathways with corresponding KO numbers.

\section{Analysis of differentially expressed genes (DEGs)}

The volcano plot of gene expression levels illustrates the distribution of up- and downregulated genes in LPN as compared with in OPN (Fig. 4a). A total of 127 DEGs were identified between LPN and OPN, and the heatmap clearly presents the clustering results of these DEGs in OPN and LPN samples, showing that genes that were significantly upregulated in LPN were significantly downregulated in OPN (Fig. 4b). Of the identified 127 DEGs, 83 were successfully annotated with BLASTX hits. Among the 83 annotated genes, 44 were upregulated and 39 were downregulated in LPN taproot, and 27 genes including 18 upregulated and 9 downregulated genes (Table S1) were assigned to specific metabolic pathways with corresponding KO numbers. Among the 18 upregulated genes, the three genes (TRINITY_DN8843_c0_g3_i1, TRINITY_DN3132_c0_g6_i1, and TRINITY_DN821_c0_g1_i1) are very likely to be related to the phenotypic difference between LPN and OPN, including two genes (TRINITY_DN8843_C0_g3_i1 and TRINITY_DN3132_C0_g6_i1) encoding enzymes originating from APX family ([EC: 1.11.1.11]) and PRX family ([EC: 1.11.1.7]), respectively, both enzymes are directly related to ROS metabolism, and one gene encoding an enzyme of the MAPK [EC: 2.7.11.24] family related to plant cell proliferation and meristematic maintenance (Table 2). Specifically, the three genes encoding APX3, PRX45 and MPK3, respectively, according to the results of BLASTP hit. In total, 47 DEGs were integrated into the protein-protein interaction (PPI) network diagram (Figure S1), including 30 upregulated (purple nodes) and 17 downregulated DEGs (green nodes), with five genes involved in ATP binding. Moreover, PPI networks illustrate that both gene (TRINITY_DN8843_c0_g3_i1) encoding APX3 and gene 
(TRINITY_DN821_c0_g1_i1) encoding MPK3 directly interact with gene TRINITY_DN4949_c0_g2_i2, as shown by the two straight lines among the three purple nodes in a red circle (Figure S1). GO annotation results showed that the molecular function of gene TRINITY_DN4949_c0_g2_i2 was implicated in ATP binding. This suggests that APX3 and MPK3 are also indirectly involved in the energy metabolism of PN root cells.

Table 2 Pathway assignments of enzymes encoded by the three upregulated genes in LPN.

\begin{tabular}{|c|c|c|c|c|c|}
\hline $\begin{array}{l}\text { Enzyme } \\
\text { family }\end{array}$ & Definition & $\begin{array}{l}\text { Enzyme } \\
\text { name }\end{array}$ & A. thaliana gene & LFC & Padj \\
\hline $\begin{array}{l}\text { [EC: } \\
1.11 .1 .11]\end{array}$ & L-Ascorbate peroxidase & APX3 & AT4G35000 & 4.56 & $9.42 \mathrm{E}-06$ \\
\hline $\begin{array}{l}\text { [EC: } \\
\text { 1.11.1.7] }\end{array}$ & Class III peroxidase & PRX45 & AT4G30170 & 3.73 & 0.001047 \\
\hline $\begin{array}{l}\text { [EC: } \\
2.7 .11 .24]\end{array}$ & $\begin{array}{l}\text { Mitogen-activated protein } \\
\text { kinase }\end{array}$ & MPK3 & AT3G45640 & 2.92 & $1.21 \mathrm{E}-08$ \\
\hline
\end{tabular}

Abbreviations: LFC, $\log _{2}$ (fold change); Padj, adjusted $P$ value

Measurement results of $\mathrm{H}_{2} \mathrm{O}_{2}$ content and enzyme activity

APX and PRX enzyme activities and $\mathrm{H}_{2} \mathrm{O}_{2}$ concentrations were measured in OPN and LPN. The $\mathrm{H}_{2} \mathrm{O}_{2}$ concentrations in OPN and LPN were $3.18 \pm 0.70$ and $2.50 \pm 0.80$ ( $\mu \mathrm{mol} / \mathrm{g}$ fresh weight), respectively (Fig. 5a). The statistical results showed that there was a significant difference in the $\mathrm{H}_{2} \mathrm{O}_{2}$ content between OPN and LPN $(p<0.05)$. The enzyme activities of APX and PRX in OPN were 3.85 \pm 0.64 and $1837.08 \pm 556.74$ (U/g fresh weight), and those in LPN taproots were $4.79 \pm 0.75,3177.54 \pm$ 1522.90 (U/g fresh weight), respectively. The statistical results showed that the activities of the two antioxidant enzymes were significantly upregulated in LPN compared with in OPN $(p<0.05)$ (Fig. 5b and 5c). Thus, upregulated APX and PRX enzyme activities led to lower $\mathrm{H}_{2} \mathrm{O}_{2}$ content in LPN taproots.

\section{RT-qPCR analysis results}

The fragments per kilobase million (FPKM) values of the three genes encoding APX3, PRX45, and MPK3 in OPN were $0.31 \pm 0.13,0.44 \pm 0.37$, and $0.93 \pm 0.16$, whereas those in LPN were $7.43 \pm 1.89,5.20 \pm 1.40$, and $14.00 \pm 4.10$, respectively. The statistical results of FPKM values showed that the relative expression levels of the three genes encoding APX3, PRX45, and MPK3 in LPN were significantly higher than in OPN $(p<0.05)$ (Fig. 5d). The relative expression levels of the three genes in OPN calculated based on RT-qPCR were $1.03 \pm 0.31,1.01 \pm 0.22$, and $1.03 \pm 0.25$, whereas those in LPN were $2.89 \pm 0.45,4.48 \pm 0.26$ and $2.94 \pm 0.50$, respectively. Interestingly, the relative expression levels of the three genes in LPN were approximately 3-, 4-, and 3-fold higher than those in OPN, respectively. Thus, the overall relative expression levels of genes encoding APX3, PRX45, and MPK3 were significantly higher in LPN than in OPN $(p<0.01)$ (Fig. 5e). 
Both RT-qPCR and statistical analyses results of FPKM values confirmed the analysis of DEGs; that is, the relative expression levels of the three genes encoding MPK3, APX3, and PRX45 were upregulated in LPN.

\section{Discussion}

ROS are extremely active chemical substances and mainly exist in two forms: radicals with free electrons such as superoxide $\left(\mathrm{O}_{2}^{-}\right)$and hydroxyl radical $\left(\mathrm{OH}^{\circ}\right)$ and nonradicals such as hypochlorous acid $(\mathrm{HOCl})$ and hydrogen peroxide $\left(\mathrm{H}_{2} \mathrm{O}_{2}\right)$ [55]. Different types of ROS have different chemical properties; $\mathrm{H}_{2} \mathrm{O}_{2}$ is the most stable ROS molecule [56, 57]. Depending on the type and concentration of ROS produced in the cells, different physiological reactions may occur. ROS can induce the expression of stress-responsive genes at low concentrations but can lead to oxidative damage to important biomacromolecules such as lipids, nucleic acids, and proteins, eventually causing cell death at high concentrations $[58,59]$. To date, many studies have reported important roles of ROS, including regulation of growth throughout plant root development, maintenance of apical meristem, and promotion of root elongation; the related internal molecular mechanisms have also been explored [24]. Specifically, the molecular mechanism of ROSmediated control of the cell cycle, including cellular proliferation, elongation, and differentiation, has been widely demonstrated in $A$. thaliana and other plants [25-30,55].

Plant cell expansion and elongation are mostly determined by the plasticity and structure of the cell wall (CW). The cellular expansion rate, namely cellular dynamics, is closely associated with the balance between CW stiffening and loosening, both of which are controlled by ROS metabolic processes [26]. Thus, ROS production and scavenging are directly related to the elongation and development of plant roots. ROS is generated mainly by the PRX and NADPH oxidase families [26, 33], whereas its scavenging is mainly regulated by antioxidant enzymes such as CAT, APX, PRX, SOD, and some nonenzymatic antioxidants, such as reduced glutathione and ascorbic acid [34,38]. A number of early studies on $A$. thaliana have confirmed that accumulation of $\mathrm{H}_{2} \mathrm{O}_{2}$ in plant root cells can enhance $\mathrm{CW}$ rigidity to hinder cellular elongation and proliferation by repressing the expression of cell cycle-related genes CyclinB and CyclinD, thus leading to shorter roots. In contrast, scavenging an excess of $\mathrm{H}_{2} \mathrm{O}_{2}$ in plant root cells contributes to maintenance of intracellular redox homeostasis in favor of cellular proliferation rather than differentiation, thus leading to the development of longer roots $[21,23,26,28]$. In the present study, it was found that the $\mathrm{H}_{2} \mathrm{O}_{2}$ concentration in LPN was significantly lower than that in OPN $(p<0.05)$ (Fig. 5a). As shown by green arrows in Fig. 6 , low $\mathrm{H}_{2} \mathrm{O}_{2}$ concentration in root cell, which is conducive to the expression of cell cycle-related genes CyclinB and CyclinD, to promote cell division and root growth. Therefore, it is possible that the emergence of the LPN phenotype is tightly correlated with lower $\mathrm{H}_{2} \mathrm{O}_{2}$ concentration caused by the high expression of some antienzymes in root cells.

APXs, bifunctional enzymes with CAT and broad-spectrum peroxidase activity, are the core enzymes that scavenge ROS in plants $[60,61]$. In plant cells, APXs scavenge $\mathrm{H}_{2} \mathrm{O}_{2}$, which mainly participates in the first step of the ascorbate-glutathione cycle, catalyzing the reaction of L-ascorbate $+\mathrm{H}_{2} \mathrm{O}_{2}+2 \mathrm{H}^{+}=\mathrm{L}$ ascorbate + L-dehydroascorbate $+2 \mathrm{H}_{2} \mathrm{O}$ to convert excess $\mathrm{H}_{2} \mathrm{O}_{2}$ into $\mathrm{H}_{2} \mathrm{O}$ [62]. During plant development, 
an increasing number of APX-encoding genes, such as APX1-6, were detected in $A$. thaliana, and their activity and function have been further confirmed [63]. In addition, other APX-encoding genes have been found in rice, wheat, and potato tubers. A number of studies on the effects of APX-knockout genes on plant physiological functions, growth processes, and antioxidant metabolism suggest that APXs can influence plant growth and development by regulating cellular redox signaling pathways involved in plant growth [64-68]. The up-regulated expression of gene (TRINITY_DN8843_c0_g3_i1) encoding APX3 in LPN was validated by RT-qPCR results (Fig. 5e) and APX enzyme activity analysis (Fig. 5b) in this study. Based on the known function of APX3, up-regulated APX3 also confirmed the lower content of $\mathrm{H}_{2} \mathrm{O}_{2}$ in LPN compared with in OPN. Therefore, it is reasonable to infer that the up-regulated APX3 in LPN can maintain the content of $\mathrm{H}_{2} \mathrm{O}_{2}$ in cells at a lower level compared with that in OPN, increase the rate of cell proliferation, and promote root elongation (green arrows in Fig. 6)

PRXs are plant CW-localized proteins that have been shown to be involved in plant CW dynamics [69]. PRXs can generate ROS such as $\cdot \mathrm{OH}$ and $\mathrm{HOO} \cdot$ to promote cell elongation in the hydroxylic cycle and oxidize various substrates to polymerize typical $\mathrm{CW}$ lignin compounds to stiffen the $\mathrm{CW}$ in the peroxidative cycle; both cycles are capable of regulating the $\mathrm{H}_{2} \mathrm{O}_{2}$ level $[26,70]$. To be specific, during the peroxidative cycle, some PRXs can oxidize molecules such as monolignols, suberin units, and ferulic acids linked to diverse polymers to form impregnable cross-links between $\mathrm{CW}$ polymers and proteins in $\mathrm{CW}$ network, leading to a tight $\mathrm{CW}$, which directly reduces cellular elongation and expansion capacity [23, 71]. However, during the hydroxylic cycle, other PRXs with extremely strong activity can produce $\cdot \mathrm{OH}$ and $\mathrm{HOO}$, which can nonspecifically break polysaccharide covalent bonds in various types of organic molecules to enhance nonenzymatic CW loosening and consequently promote cellular elongation [26]. A previous study on $A$. thaliana confirmed that the expression inhibition of genes encoding PRXs results in an increase in $\mathrm{H}_{2} \mathrm{O}_{2}$ content, whereas up-regulated expression of genes encoding PRXs can continually scavenge excess $\mathrm{H}_{2} \mathrm{O}_{2}$ in plant root cells and maintain intracellular redox homeostasis in favor of cellular proliferation rather than differentiation, leading to the development of longer roots [21]. Briefly, the function of PRXs can be classified into two main categories: stiffening and loosening CW. Additionally, based on previous studies of multiple phenotypes of the corresponding AtPrx-deficient mutants of $A$. thaliana (such as mutants deficient in AtPrx02/25/71, AtPrx33/34, and AtPrx53), one PRX category, including AtPrx37 [72], AtPrx02/25/71 [73], AtPrx64 [74], and AtPrx72 [75], can promote the hardening of the plant $\mathrm{CW}$ by taking $\mathrm{H}_{2} \mathrm{O}_{2}$ as an electron acceptor to catalyze lignin formation and polymerization [7678]. In other words, they can catalyze lignin formation and polymerization to stiffen the plant $\mathrm{CW}$ in the presence of $\mathrm{H}_{2} \mathrm{O}_{2}$, thus leading to shorter roots. Other categories of PRXs, including AtPrx36, AtPrx39, AtPrx40, AtPrx57, AtPrx33/34, and AtPrx53, were found to be closely associated with CW loosening and root elongation $[22,79,80]$. Specifically, $A$. thaliana with overexpression of gene encoding PRX34 had longer roots than the wild type (WT), whereas the plants with double knockdown of genes encoding PRX33 and PRX34 had shorter roots than the WT. The gene identified in this study, which encodes PRX45 (namely AtPrx45), was significantly upregulated in LPN compared with in OPN (Fig. 5c). PRX45, like other PRXs, mainly exists in roots and hypocotyl tissues and acts in response to oxidative stress. However, the specific molecular regulation mechanism of PRX45 in the plant root CW has not been reported. The up- 
regulated expression of the gene (TRINITY_DN3132_C0_g6_i1) encoding PRX45 in LPN was validated by RT-qPCR results (Fig. 5e) and PRX enzyme activity analysis (Fig. 5c). It is reasonable to speculate that PRX45 may participate in the hydroxylic cycle to produce $\cdot \mathrm{OH}$ and $\mathrm{HOO} \cdot$ and split glycosidic bonds or certain covalent bonds in CW components, resulting in cell elongation and expansion, and eventually root elongation (blue arrow in Fig. 6). The specific molecular function of PRX45 may be comprehensively confirmed in A. thaliana roots, similar to other AtPrx-encoding genes in the foreseeable future.

MAPKs, also known as MPKs in plants, are a highly conserved enzyme family with essential functions; they are widely found in plant and animal cells. The MAPK cascade is composed of diverse proteinases involved in various biological processes, including cell proliferation, differentiation, response to diverse stresses, and tolerance to environmental stimuli [39-41]. For instance, it has been shown that the activity of PsMPK2 in pea and ZmMPK5 in maize can be elevated by $\mathrm{H}_{2} \mathrm{O}_{2}$ stress $[81,82]$. TaMPK4 plays a critical role in mediating plant tolerance to various stresses by inducing root growth and regulating cellular ROS metabolism [83]. StMAPK11 upregulation can enhance CAT and PRX activity to increase the antioxidant activity in potato, tobacco, and $A$. thaliana [84]. The activities of MPK3 and MPK6 in $A$. thaliana are positively correlated with plant defense against oxidative stress triggered by salt stress [85]. Importantly, the regulatory functions of the MAPK cascade in plant shoot apical meristem (SAM) have long been proposed, as MPK3 and MPK6 are crucial regulators of stem cell homeostasis in $A$. thaliana by participating in CLAVATA peptide receptor-WUSCHEL transcription factor (CLV-WUS) signaling pathways of SAM development $[86,87]$. In addition, two recently reported studies indicated that MPK3 can positively regulate root meristem growth factor 1 (RGF1)-mediated root growth and development and promote cell division in the root apical meristem, leading to plant root elongation $[88,89]$. In the present study, RT-qPCR was conducted to validate the expression of the gene encoding MPK3 in LPN and OPN. The validation results were consistent with the analysis of DEGs; the gene (TRINITY_DN821_c0_g1_i1) encoding MPK3 was upregulated in LPN as compared with in OPN (Fig. 5e). Based on recent studies on MPK3 function in $A$. thaliana, it is rational to assume that up-regulated gene encoding MPK3 is more favorable for stem cell maintenance of plant root meristem and can mediate RGF1 expression, leading to promotion of cell proliferation in the PN taproot. Therefore, up-regulated MPK3 activity is likely to be one of the driving forces for the formation of the LPN phenotype (purple arrows in Fig. 6).

Additionally, previous studies have confirmed that plant root development is jointly determined by the rates of cell proliferation and the extent of cell elongation [30,90]. Several published studies on different sweet potato root types suggest that up-regulated antioxidant enzyme levels could improve plant root growth and development, and under certain circumstances, may increase yield [26, 33, 91]. In the present study, DEGs analysis showed that the genes encoding APX3, PRX45, and MPK3 were significantly upregulated in LPN as compared with in OPN. Further, $\mathrm{H}_{2} \mathrm{O}_{2}$ content, APX and PRX enzyme activity, and RT-qPCR analyses in LPN and OPN further verified the transcriptome analysis. This illustrates that APX3, PRX45, and MPK3 may be directly or indirectly involved in the process of promoting PN taproot development and elongation. A hypothetical molecular regulatory network leading to LPN formation may be based on the joint interference of $\mathrm{H}_{2} \mathrm{O}_{2}$, APX3, PRX45, and MPK3 (Fig. 6). 


\section{Conclusion}

In this study, we performed de novo transcriptome assembly and functional annotation from six PN taproot samples (three each of LPN and OPN) and determined the causes of phenotypic differences in the development process of PN by analyzing DEGs of LPN and OPN. DEGs analysis showed that genes encoding APX3, PRX45, and MPK3 were significantly upregulated in LPN compared with in OPN. These three enzymes play pivotal roles in ROS metabolism and oxidative stress. Many previous studies have demonstrated that the process of ROS metabolism is closely related to the proliferation, elongation, and differentiation of plant root cells. It has recently been reported that MPK3 can positively regulate RGF1mediated root growth and is indispensable for stem cell maintenance in the shoot apical stem of $A$. thaliana.

In summary, we confirmed that the PN taproot phenotype is influenced by a network controlling ROS metabolism during the taproot development process. Based on the results of this study and those of previously published studies on the relationship between ROS metabolism and plant root development, it can be concluded that the taproot phenotype of LPN is due to the upregulated expression of genes encoding APX3, PRX45, and MPK3. The results of this study provide a reliable explanation for the phenotypic differences between OPN and LPN, and they offer further insights into the genetic mechanism of phenotypic differences for other species of the Panax genus. Our results will be useful for future molecular breeding of PN.

\section{Declarations}

\section{Acknowledgments}

Not applicable.

\section{Authors' Contributions}

LY performed Experiment section, data analysis, and manuscript writing. YL provided guidance for data analysis and manuscript revision. XC and LH conceived the initial idea and provided scientific advice for the paper. HW and PW provided assistance for the experiment section and use of analysis software. MG assist with sample collection.

Funding: This study was financially supported by the National Natural Science Foundation of China (Grant No. 31960134), the Ability Establishment of Sustainable Use for Valuable Chinese Medicine Resources (Grant No. 2060302), and the Major Science and Technology Special Project of Yunnan Province (Grant No. 202102AA310034).

\section{Availability of data and materials}

The datasets supporting the conclusion of this article are included within the article and its additional files, and the sequencing data for all samples is available in the NCBI database with the BioProject 
number: PRJNA762619 (SRA: SRR15882016), [unique persistent identifier and hyperlink to datasets in https://www.ncbi.nlm.gov/search/all/?term=SRR15882016].

\section{Declarations}

\section{Ethics approval and consent to participate}

The Panax notoginseng plants used in this study were kindly provided by Wenshan University, and the collected samples for plant research use only. The use of Panax notoginseng plants in this study is compiled with the relevant institutional, national, and international guidelines and legislation.

\section{Consent for publication}

Not applicable.

\section{Competing interests}

The authors declare no conflict of interest.

\section{Author details}

1 Faculty of Life Science and Technology, Kunming University of Science and Technology, 650000 Kunming, China

2 Wenshan University, 663000 Wenshan, China

3 National Resource Center for Chinese Materia Medica, Chinese Academy of Chinese Medical Sciences, 100700 Beijing, China

${ }^{4}$ Key Laboratory of Panax notoginseng Resources Sustainable Development and Utilization of State Administration of Traditional Chinese Medicine, 650000 Kunming, China

5 Yunnan Provincial Key Laboratory of Panax notoginseng, 650000 Kunming, China

${ }^{6}$ Kunming Key Laboratory of Sustainable Development and Utilization of Famous-Region Drug, 650000 Kunming, China

7 Sanqi Research Institute of Yunnan Province, 650000 Kunming, China

*Correspondence: liuyuan513@kust.edu.cn; sanqi37@vip.sina.com

\section{References}

1. Xu CC, Wang WW, Wang B, Zhang T, Cui XM, Pu YQ, Li N. Analytical methods and biological activities of Panax notoginsengsaponins: Recent trends.J Ethnopharmacol.2019; 236: 443-465. 
2. Wang T, Guo RX, Zhou GH, Zhou XD, Kou ZZ, Sui F, Li C, Tang LY, Wang ZJ. Traditional uses, botany, phytochemistry, pharmacology and toxicology of Panax notoginseng (Burk.) FH Chen: A review.J. Ethnopharmacol.2016; 188: 234-258.

3. Kim DH. Chemical Diversity of Panax ginseng, Panax quinquifolium, and Panax notoginseng. $J$ Ginseng Res.2012; 36: 1-15.

4. Duan L, Xiong XJ, Hu JY, Liu YM, Li J, Wang J. Panax notoginseng saponins for treating coronary artery disease: A functional and mechanistic overview.Front Pharmacol.2017; 8: 702.

5. Hu S, Liu T, Wu Y, Yang W, Hu S, Sun Z, Li P, Du S. Panax notoginsengsaponins suppress lipopolysaccharide-induced barrier disruption and monocyte adhesion on bEnd. 3 cells via the opposite modulation of Nrf2 antioxidant and NF-KB inflammatory pathways. Phytotherapy Res.2019; 33: 31633176.

6. Yang ZJ, Liu GZ, Zhang GH, Yan J, Dong Y, Lu YC, Fan W, Hao B, Lin Y, Li Y,et al. The chromosome-scale high-quality genome assembly of Panax notoginseng provides insight into dencichine biosynthesis. Plant Biotechnol J.2021; 19: 869-871.

7. Jiang Z, Tu L, Yang W, Zhang Y, Hu T, Ma B, Lu Y, Cui X, Gao J, Wu X,et al. The chromosome-level reference genome assembly for Panax notoginsengand insights into ginsenoside biosynthesis. Plant Communications.2021; 2: 100113.

8. Fan GY, Liu XC, Sun S, Shi CC, Du X, Han K, Yang BR, Fu YY, Liu MH, Seim l,et al. The chromosome level genome and genome-wide association study for the agronomic traits of Panax notoginseng. Iscience.2020; 23: 101538.

9. Zhang D, Li W, Chen ZJ, Wei FG, Liu YL, Gao LZ. SMRT- and Illumina-based RNA-seq analyses unveil the ginsinoside biosynthesis and transcriptomic complexity in Panax notoginseng.Sci Rep.2020; 10: 15310.

10. Zhang D, Li W, Xia EH, Zhang QJ, Liu Y, Zhang Y, Tong Y, Zhao Y, Niu YC, Xu JH, et al. The medicinal herb Panax notoginseng genome provides insights into ginsenoside biosynthesis and genome evolution.Mol Plant.2017; 10: 903-907.

11. Chen W, Kui L, Zhang GH, Zhu SS, Zhang J, Wang X, Yang M, Huang HC, Liu YX, Wang Y,et al. Wholegenome sequencing and analysis of the Chinese Herbal plant Panax notoginseng. Mol Plant.2017; 10: 899-902.

12. Zhang K, Sun C, Hu YX, Yang JY, Wu CF. Network pharmacology reveals pharmacological effect and mechanism of Panax notoginseng (Burk.) F. H. Chen on reproductive and genetic toxicity in male mice.J Ethnopharmacol.2021; 270: 113792. 
13. Yang BW, Hahm YT. Transcriptome analysis using de novo RNA-seq to compare ginseng roots cultivated in different environments. Plant Growth Regul.2018; 84: 149-157.

14. Wachsman G, Sparks EE, Benfey PN. Genes and networks regulating root anatomy and architecture. New Phytol.2015; 208: 26-38.

15. Novoplansky A. What plant roots know? SeminCellDev Biol.2019; 92: 126-133.

16. Yang M, Zhu L, Pan C, Xu L, Liu Y, Ke W, Yang P. Transcriptomic analysis of the regulation of rhizome formation in temperate and tropical lotus (Nelumbo nucifera).Sci Rep.2015; 5: 13059.

17. Li XJ, Yang JL, Hao B, Lu YC, Qian ZL, Li Y, Ye S, Tang JR, Chen M, Long GQ, et al. Comparative transcriptome and metabolome analyses provide new insights into the molecular mechanisms underlying taproot thickening in Panax notoginseng.BMC Plant Biol.2019; 19: 451.

18. Xanthopoulou A, Ganopoulos I, Psomopoulos F, Manioudaki M, Moysiadis T, Kapazoglou A, Osathanunkul M, Michailidou S, Kalivas A, Tsaftaris A, et al. De novo comparative transcriptome analysis of genes involved in fruit morphology of pumpkin cultivars with extreme size difference and development of EST-SSR markers. Gene.2017; 622: 50-66.

19. Pal T, Malhotra N, Chanumolu SK, Chauhan RS. Next-generation sequencing (NGS) transcriptomes reveal association of multiple genes and pathways contributing to secondary metabolites accumulation in tuberous roots of Aconitum heterophyllum Wall. Planta.2015; 242: 239-258.

20. Gu N, Zhang X, Gu X, Zhao L, Godana EA, Xu M, Zhang H: Transcriptomic and proteomic analysis of the mechanisms involved in enhanced disease resistance of strawberries induced by Rhodotorula mucilaginosa cultured with chitosan. Postharvest Biol Technol.2021;172: 111355.

21. Tsukagoshi H, Busch W, Benfey PN. Transcriptional regulation of ROS controls transition from proliferation to differentiation in the root. Cell.2010; 143: 606-616.

22. Passardi F, Tognolli M, De Meyer M, Penel C, Dunand C. Two cell wall associated peroxidases fromArabidopsis influence root elongation. Planta.2006; 223: 965-974.

23. Dunand C, Crèvecoeur M, Penel C. Distribution of superoxide and hydrogen peroxide in Arabidopsis root and their influence on root development: possible interaction with peroxidases. New Phytol.2007; 174: 332-341.

24. Eljebbawi A, Guerrero YdCR, Dunand C, Estevez JM. Highlighting reactive oxygen species as multitaskers in root development. iScience.2021; 24: 101978.

25. Schopfer P. Hydroxyl radical-induced cell-wall loosening in vitro and in vivo: implications for the control of elongation growth.Plant J.2001; 28: 679-688. 
26. Francoz E, Ranocha P, Nguyen-Kim H, Jamet E, Burlat V, Dunand C: Roles of cell wall peroxidases in plant development. Phytochemistry.2015; 112: 15-21.

27. Chapman JM, Muhlemann JK, Gayomba SR, Muday GK: RBOH-dependent ROS synthesis and ROS scavenging by plant specialized metabolites to modulate plant development and stress responses.Chem. Res. Toxicol.2019; 32: 370-396.

28. Schippers JHM, Foyer CH, van Dongen JT. Redox regulation in shoot growth, SAM maintenance and flowering. Curr Opin Plant Biol.2016; 29: 121-128.

29. Wrzaczek M, Brosché M, Kangasjärvi J. ROS signaling loops - production, perception, regulation.Curr Opin Plant Biol.2013; 16: 575-582.

30. Tsukagoshi H. Defective root growth triggered by oxidative stress is controlled through the expression of cell cycle-related genes. Plant Sci.2012; 197: 30-39.

31. Tognetti VB, Bielach A, Hrtyan M: Redox regulation at the site of primary growth: auxin, cytokinin and ROS crosstalk.Plant Cell Environ.2017; 40: 2586-2605.

32. Gechev T, Petrov V. Reactive oxygen species and abiotic stress in plants. Int J Mol Sci.2020; 21: 7433.

33. Orman-Ligeza B, Parizot B, de Rycke R, Fernandez A, Himschoot E, Van Breusegem F, Bennett MJ, Périlleux $\mathrm{C}$, Beeckman T, Draye X. RBOH-mediated ROS production facilitates lateral root emergence in Arabidopsis. Development.2016; 143: 3328-3339.

34. Choudhary A, Kumar A, Kaur N. ROS and oxidative burst: Roots in plant development. Plant Diversity.2020; 42: 33-43.

35. Das K, Roychoudhury A. Reactive oxygen species (ROS) and response of antioxidants as ROSscavengers during environmental stress in plants. Front Environ Sci.2014; 2: 53.

36. Corpas FJ, Barroso JB, Palma JM, Rodriguez-Ruiz M. Plant peroxisomes. A nitro-oxidative cocktail. Redox Biol.2017, 11: 535-542.

37. Yao M, Ge W, Zhou Q, Zhou X, Luo M, Zhao Y, Wei B, Ji S. Exogenous glutathione alleviates chilling injury in postharvest bell pepper by modulating the ascorbate-glutathione (AsA-GSH) cycle.Food Chem.2021; 352: 129458.

38. Eltayeb AE, Kawano N, Badawi GH, Kaminaka H, Sanekata T, Shibahara T, Inanaga S, Tanaka K. Overexpression of monodehydroascorbate reductase in transgenic tobacco confers enhanced tolerance to ozone, salt and polyethylene glycol stresses. Planta.2007; 225: 1255-1264.

39. Pitzschke A, Hirt H. Disentangling the complexity of mitogen-activated protein kinases and reactive oxygen species signaling.Plant Physiol.2009; 149: 606-615. 
40. Shin HY, You MK, Jeung JU, Shin JS. OsMPK3 is a TEY-type rice MAPK in group C and phosphorylates OsbHLH65, a transcription factor binding to the E-box element.Plant Cell Rep.2014; 33: 1343-1353.

41. Moustafa K, AbuQamar S, Jarrar M, Al-Rajab AJ, Trémouillaux-Guiller J. MAPK cascades and major abiotic stresses. Plant Cell Rep.2014; 33: 1217-1225.

42. Bolger AM, Lohse M, Usadel B. Trimmomatic: a flexible trimmer for Illumina sequence data. Bioinformatics.2014; 30: 2114-2120.

43. Haas BJ, Papanicolaou A, Yassour M, Grabherr M, Blood PD, Bowden J, Couger MB, Eccles D, Li B, Lieber M.et al. De novo transcript sequence reconstruction from RNA-seq using the Trinity platform for reference generation and analysis. Nat Protoc.2013; 8: 1494-1512.

44. Fu LM, Niu BF, Zhu ZW, Wu ST, Li WZ. CD-HIT: accelerated for clustering the next-generation sequencing data. Bioinformatics.2012; 28: 3150-3152.

45. Ashburner M, Ball CA, Blake JA, Botstein D, Butler H, Cherry JM, Davis AP, Dolinski K, Dwight SS, Eppig JT,et al. Gene Ontology: tool for the unification of biology.Nat Genet.2000; 25: 25-29.

46. Kanehisa M, Goto S, Sato Y, Furumichi M, Tanabe M. KEGG for integration and interpretation of largescale molecular data sets. Nucleic Acids Res.2012; 40: D109-D114.

47. Kanehisa M, Goto S. KEGG: Kyoto Encyclopedia of Genes and Genomes. Nucleic Acids Res.2000; 28: 27-30.

48. Thomas PD, Campbell MJ, Kejariwal A, Mi HY, Karlak B, Daverman R, Diemer K, Muruganujan A, Narechania A. PANTHER: A library of protein families and subfamilies indexed by function. Genome Res.2003; 13: 2129-2141.

49. Li B, Dewey CN. RSEM: accurate transcript quantification from RNA-Seq data with or without a reference genome. BMC Bioinformatics.2011; 12: 323.

50. Langmead B, Salzberg SL. Fast gapped-read alignment with Bowtie 2. Nat Methods.2012; 9: 357-359.

51. Love Ml, Huber W, Anders S. Moderated estimation of fold change and dispersion for RNA-seq data with DESeq2. Genome Biol.2014; 15: 38.

52. Young MD, Wakefield MJ, Smyth GK, Oshlack A. Gene ontology analysis for RNA-seq: accounting for selection bias. Genome Biol.2010; 11: 12.

53. Awad IAB, Rees CA, Hernandez-Boussard T, Ball CA, Sherlock G. Caryoscope: An Open Source Java application for viewing microarray data in a genomic context. BMC Bioinformatics.2004; 5: 151.

54. Tang JR, Lu YC, Gao ZJ, Song WL, Wei KH, Zhao Y, Tang QY, Li XJ, Chen JW, Zhang GH, et al. Comparative transcriptome analysis reveals a gene expression profile that contributes to rhizome 
swelling in Panax japonicus var. major. Plant Biosyst.2020; 154: 515-523.

55. Mhamdi A, Van Breusegem F. Reactive oxygen species in plant development. Development.2018; 145:dev164376.

56. Mittler R. ROS Are Good. Trends in Plant Sci.2017; 22: 11-19.

57. Waszczak C, Carmody M, Kangasjärvi J. Reactive oxygen species in plant signaling.Annu. Rev. Plant Biol. 2018, 69: 209-236.

58. Lehmann S, Serrano M, L'Haridon F, Tjamos SE, Metraux JP. Reactive oxygen species and plant resistance to fungal pathogens. Phytochemistry.2015; 112: 54-62.

59. Petrov V, Hille J, Mueller-Roeber B, Gechev TS. ROS-mediated abiotic stress-induced programmed cell death in plants. Front Plant Sci.2015; 6: 69.

60. Anjum NA, Sharma P, Gill SS, Hasanuzzaman M, Khan EA, Kachhap K, Mohamed AA, Thangavel P, Devi GD, Vasudhevan P,et al. Catalase and ascorbate peroxidase-representative $\mathrm{H}_{2} \mathrm{O}_{2}$-detoxifying heme enzymes in plants. Environ Sci Pollut Res.2016; 23: 19002-19029.

61. Pandey S, Fartyal D, Agarwal A, Shukla T, James D, Kaul T, Negi YK, Arora S, Reddy MK. Abiotic stress tolerance in plants: Myriad roles of ascorbate peroxidase.Front Plant Sci.2017; 8: 581.

62. Hasanuzzaman M, Bhuyan MHMB, Anee TI, Parvin K, Nahar K, Mahmud JA, Fujita M. Regulation of ascorbate-glutathione pathway in mitigating oxidative damage in plants under abiotic stress. Antioxidants2019; 8: 384.

63. Kuo EY, Cai M-S, Lee T-M. Ascorbate peroxidase 4 plays a role in the tolerance of Chlamydomonas reinhardtii to photo-oxidative stress. Sci Rep.2020; 10: 13287.

64. Maruta T, Sawa Y, Shigeoka S, Ishikawa T. Diversity and evolution of ascorbate peroxidase functions in chloroplasts: More than just a classical antioxidant enzyme? Plant Cell Physiol.2016; 57: 1377-1386.

65. Rosa SB, Caverzan A, Teixeira FK, Lazzarotto F, Silveira JAG, Ferreira-Silva SL, Abreu-Neto J, Margis R, Margis-Pinheiro M. Cytosolic APx knockdown indicates an ambiguous redox responses in rice. Phytochemistry.2010; 71: 548-558.

66. Secenji M, Hideg E, Bebes A, Gyoergyey J. Transcriptional differences in gene families of the ascorbate-glutathione cycle in wheat during mild water deficit. Plant Cell Rep.2010; 29: 37-50.

67. Kawakami S, Matsumoto Y, Matsunaga A, Mayama S, Mizuno M. Molecular cloning of ascorbate peroxidase in potato tubers and its response during storage at low temperature. Plant Sci.2002; 163: 829836. 
68. Andréia C, Gisele P, Barcellos RS, Werner RC, Fernanda L, Márcia M. Plant responses to stresses: role of ascorbate peroxidase in the antioxidant protection. Genet. mol biol.2012; 35: 1011-1019.

69. Cosgrove DJ. Growth of the plant cell wall. Nat Rev Mol Cell Biol.2005; 6: 850-861.

70. Passardi F, Penel C, Dunand C. Performing the paradoxical: how plant peroxidases modify the cell wall. Trends Plant Sci.2004; 9: 534-540.

71. Marzol E, Borassi C, Ranocha P, Aptekman AA, Bringas M, Pennington J, Paez-Valencia J, Pacheco JM, Rodríguez Garcia DR, del Carmen Rondón Guerrero Y,et al.Class III peroxidases PRX01, PRX44, and PRX73 potentially target extensins during root hair growth in Arabidopsis thaliana. bioRxiv.2020.02.04.932376.

72. Pedreira J, Teresa Herrera M, Zarra I, Revilla G. The overexpression of AtPrx37, an apoplastic peroxidase, reduces growth in Arabidopsis.Physiol. Plant.2011; 141: 177-187.

73. Shigeto J, Kiyonaga Y, Fujita K, Kondo R, Tsutsumi Y. Putative cationic cell-wall-bound peroxidase homologues in Arabidopsis, AtPrx2, AtPrx25, and AtPrx71, are involved in lignification. J Agric Food Chem.2013; 61: 3781-3788.

74. Lee Y, Rubio MC, Alassimone J, Geldner N. A mechanism for localized lignin deposition in the endodermis. Cell.2013; 153: 402-412.

75. Herrero J, Fernandez-Perez F, Yebra T, Novo-Uzal E, Pomar F, Angeles Pedreno M, Cuello J, Guera A, Esteban-Carrasco A, Miguel Zapata J. Bioinformatic and functional characterization of the basic peroxidase 72 from Arabidopsis thaliana involved in lignin biosynthesis. Planta.2013; 237: $1599-1612$.

76. Tsukagoshi $\mathrm{H}$. Control of root growth and development by reactive oxygen species. Curr Opin Plant Biol.2016; 29: 57-63.

77. Fujita S, De Bellis D, Edel KH, Köster P, Andersen TG, Schmid-Siegert E, Dénervaud Tendon V, Pfister A, Marhavý P, Ursache R, et al. SCHENGEN receptor module drives localized ROS production and lignification in plant roots. Embo J.2020; 39: e103894.

78. Hoffmann N, Benske A, Betz H, Schuetz M, Samuels AL. Laccases and peroxidases co-localize in lignified secondary cell walls throughout stem development.Plant physiol.2020; 184: 806-822

79. Kunieda T, Shimada T, Kondo M, Nishimura M, Nishitani K, Hara-Nishimura I. Spatiotemporal secretion of PEROXIDASE36 is required for seed coat mucilage extrusion in Arabidopsis. Plant Cell.2013; 25: $1355-1367$.

80. Jin J, Hewezi T, Baum TJ. Arabidopsis peroxidase AtPRX53 influences cell elongation and susceptibility to Heterodera schachtii.Plant signal. Behav.2011; 6: 1778-1786. 
81. Ortiz-Masia D, Perez-Amador MA, Carbonell P, Aniento F, Carbonell J, Marcote MJ. Characterization of PsMPK2, the first C1 subgroup MAP kinase from pea (Pisum sativum L.). Planta.2008; 227: 1333-1342.

82. Ding HD, Zhang XH, Xu SC, Sun LL, Jiang MY, Zhang AY, Jin YG. Induction of protection against paraquat-induced oxidative damage by abscisic acid in maize leaves is mediated through mitogenactivated protein kinase.J Integr Plant Biol.2009; 51: 961-972.

83. Hao L, Wen YL, Zhao YY, Lu WJ, Xiao K. Wheat mitogen-activated protein kinase gene TaMPK4 improves plant tolerance to multiple stresses through modifying root growth, ROS metabolism, and nutrient acquisitions. Plant Cell Rep.2015; 34: 2081-2097.

84. Zhu X, Zhang N, Liu X, Li SG, Yang JW, Hong XS, Wang FF, Si HJ. Mitogen-activated protein kinase 11 (MAPK11) maintains growth and photosynthesis of potato plant under drought condition. Plant Cell Rep.2021; 40: 491-506.

85. Lumbreras V, Vilela B, Irar S, Sole M, Capellades M, Valls M, Coca M, Pages M. MAPK phosphatase MKP2 mediates disease responses in Arabidopsis and functionally interacts with MPK3 and MPK6. Plant J.2010; 63: 1017-1030.

86. Lee H, Jun YS, Cha OK, Sheen J. Mitogen-activated protein kinases MPK3 and MPK6 are required for stem cell maintenance in the Arabidopsisshoot apical meristem. Plant Cell Rep.2019; 38: 311-319.

87. Somssich M, Je B, Simon R, Jackson D: CLAVATA-WUSCHEL signaling in the shoot meristem. Development.2016; 143: 3238-3248.

88. Lu XT, Shi HY, Ou Y, Cui YW, Chang JK, Peng L, Gou XP, He K, Li J. RGF1-RGI1, a peptide-receptor complex, regulates Arabidopsis root meristem development via a MAPK signaling cascade. Mol Plant.2020; 13: 1594-1607.

89. Shao YM, Yu XX, Xu XW, Li Y, Yuan WX, Xu Y, Mao CZ, Zhang SQ, Xu J. The YDA-MKK4/M KK5-M PK3/MPK6 cascade functions downstream of the RGF1-RGI ligand-receptor pair in regulating mitotic activity in root apical meristem. Mol Plant.2020; 13: 1608-1623.

90. Beemster GTS, Baskin TI. Analysis of cell division and elongation underlying the developmental acceleration of root growth in Arabidopsis thaliana. Plant Physiol.1998; 116: 1515-1526.

91. Kim YH, Park SC, Ji CY, Lee JJ, Jeong JC, Lee HS, Kwak SS. Diverse antioxidant enzyme levels in different sweetpotato root types during storage root formation.Plant Growth Regul.2015; 75: 155-164.

\section{Figures}



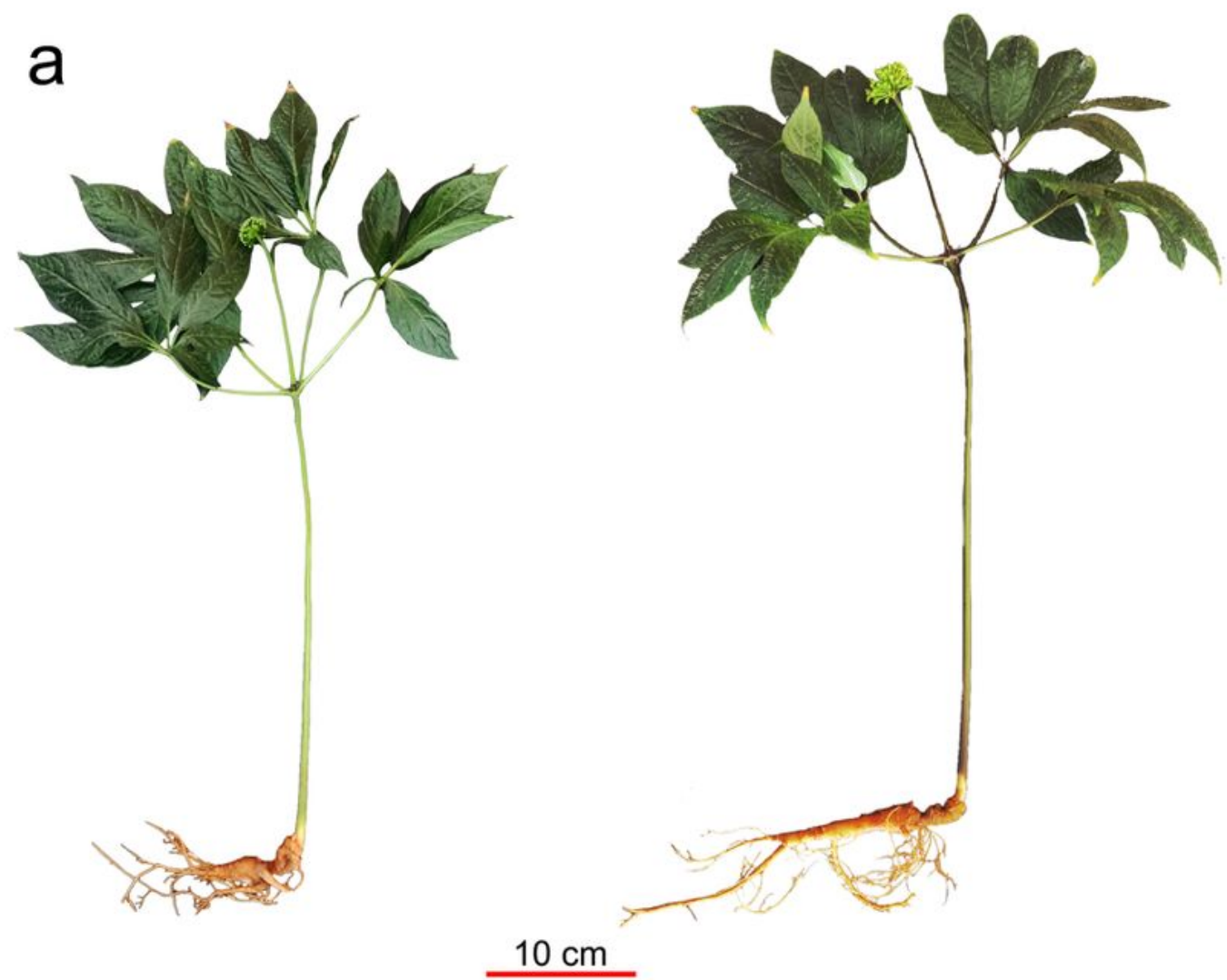

b

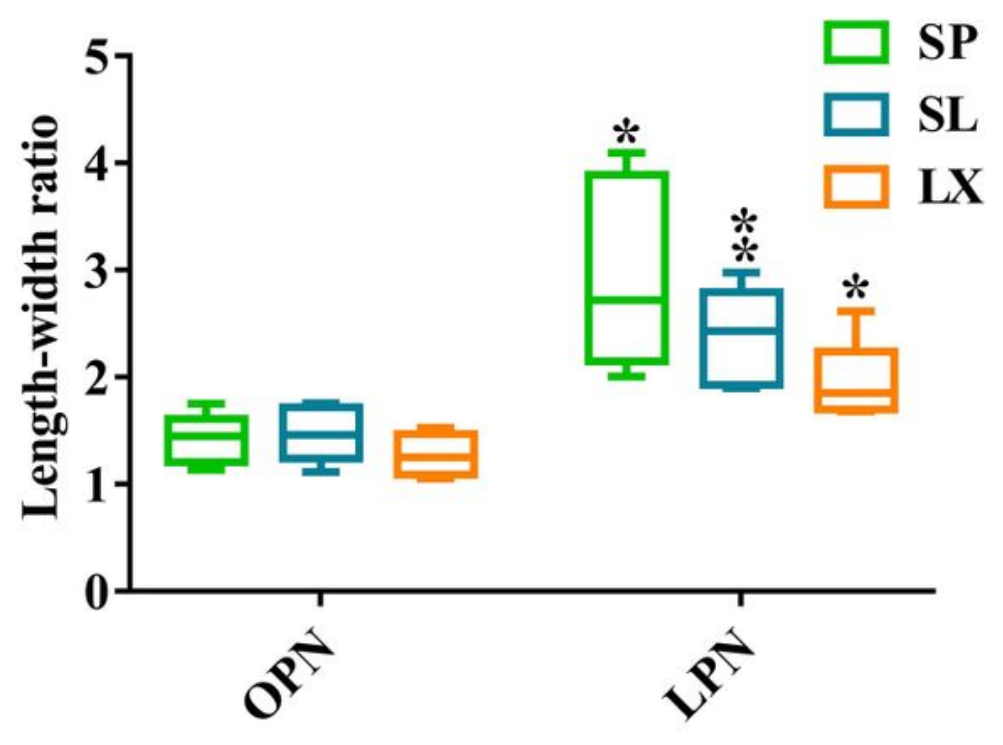

Figure 1

Phenotype characteristics of OPN and LPN taproots. (a) The typical phenotypes of OPN (left) and LPN taproot (right), scale bar: $10 \mathrm{~cm}$. (b) The statistical result of length-width ratios of OPN and LPN taproots. LX, SL, and SP represent Luxi county, Shilin county, and Shipin county, respectively. Data are presented as mean \pm s.d. $(n=5)$. The asterisk and double asterisks represent significant difference determined by the independent-sample t-test in PASW at $p<0.05\left(^{(}\right)$and $p<0.01(* *)$, respectively. 


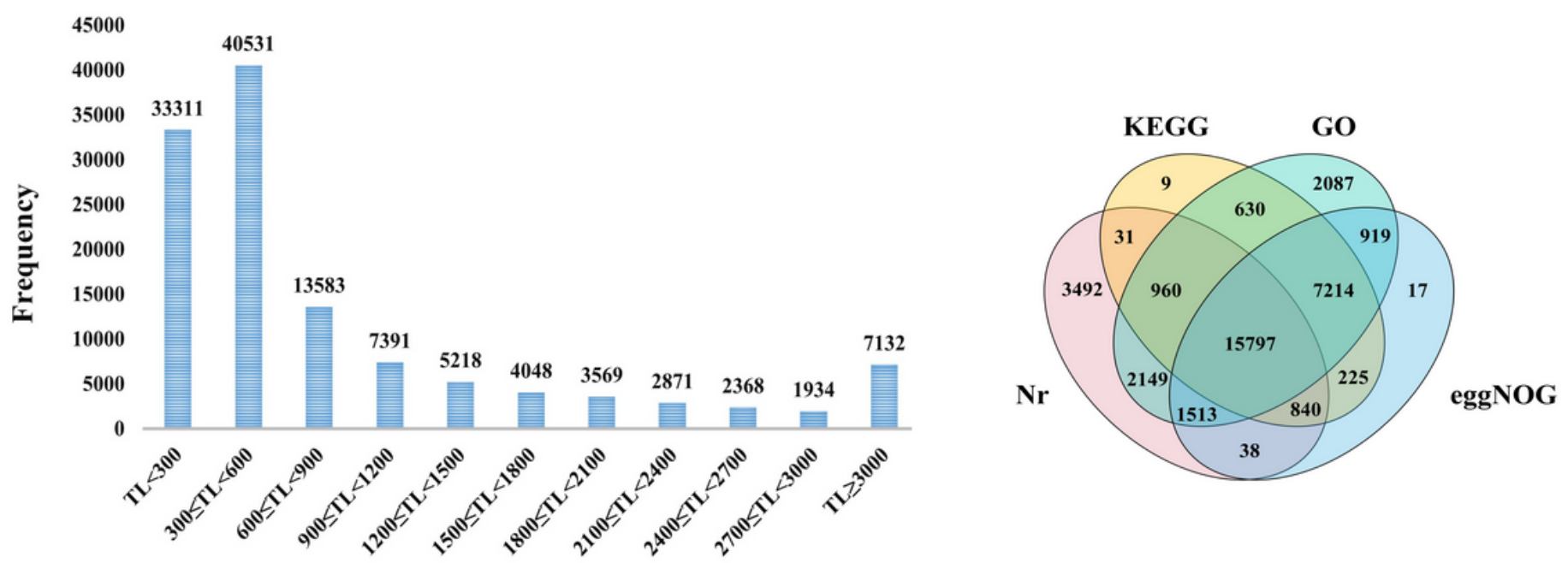

Length interval of transcripts

Figure 2

Statistical results of length distribution and database annotation of the reference transcriptome. (a) The transcript length ( $T L$ ) distribution of all transcripts. (b) The annotation result statistics for all transcripts annotated to the four databases, namely Nr, KEGG, GO and eggNOG.

$\mathbf{a}$

Cellular component

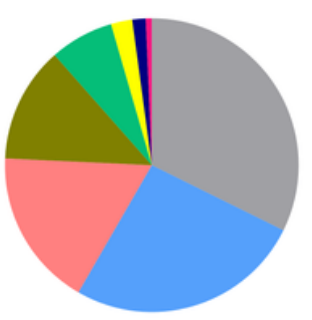

- membrane (31.03\%)

- organelle $(24.99 \%)$

nucleus $(16.71 \%)$

- cytoplasm (12.24\%)

- supramolecular complex $(6.75 \%)$

extracellular region (2.25\%)

- cell wall $(1.41 \%)$

- peroxisome $(0.64 \%)$

b

Biological process

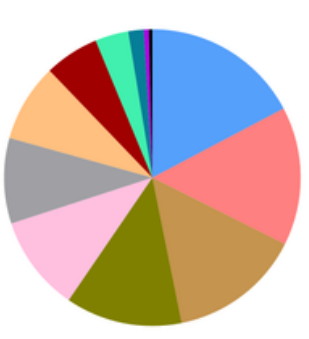

C

\section{Molecular function}

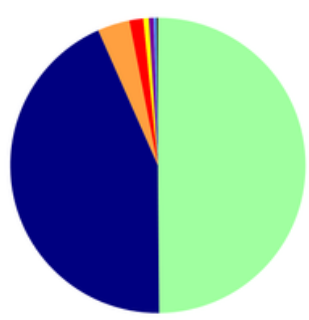

binding (49.01\%)

- ase activity $(\mathbf{4 2 . 7 6 \% )}$

- transporter activity $(3.45 \%)$

- structural molecule activity $(1.51 \%)$

- calcium channel activity $(0.58 \%)$

- translation regulator activity $(\mathbf{0 . 5 2} \%)$

- transcriptional regulator factor $(0.30 \%)$

- nutrient reservoir activity $(0.14 \%)$ d

$$
\begin{aligned}
& \text { metabolic process }(16.31 \%) \\
& \text { biological regulation }(14.26 \%) \\
& \text { response to stimulus }(13.61 \%) \\
& \text { signal transduction and transport }(11.20 \%) \\
& \text { DNA process }(9.87 \%) \\
& \text { protein process }(8.79 \%) \\
& \text { RNA process }(8.03 \%) \\
& \text { development process }(5.60 \%) \\
& \text { cell cycle }(3.43 \%) \\
& \text { ubiquitination }(1.60 \%) \\
& \text { multicellular organismal process }(0.52 \%) \\
& \text { localization }(0.35 \%)
\end{aligned}
$$

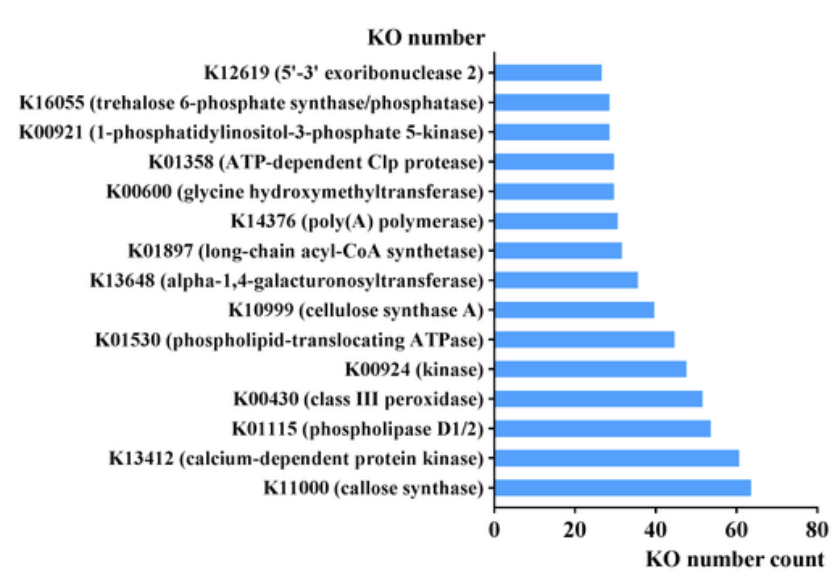




\section{Figure 3}

The GO classification results of annotated unigenes and the top $15 \mathrm{KO}$ numbers. Pie charts indicate the percentage of genes classified into diverse GO-slim terms for (a) cellular components, (b) biological processes, and (c) molecular functions. (d) the statistics of the top $15 \mathrm{KEGG}$ Orthology (KO) numbers mapped to the KEGG database.

a

b
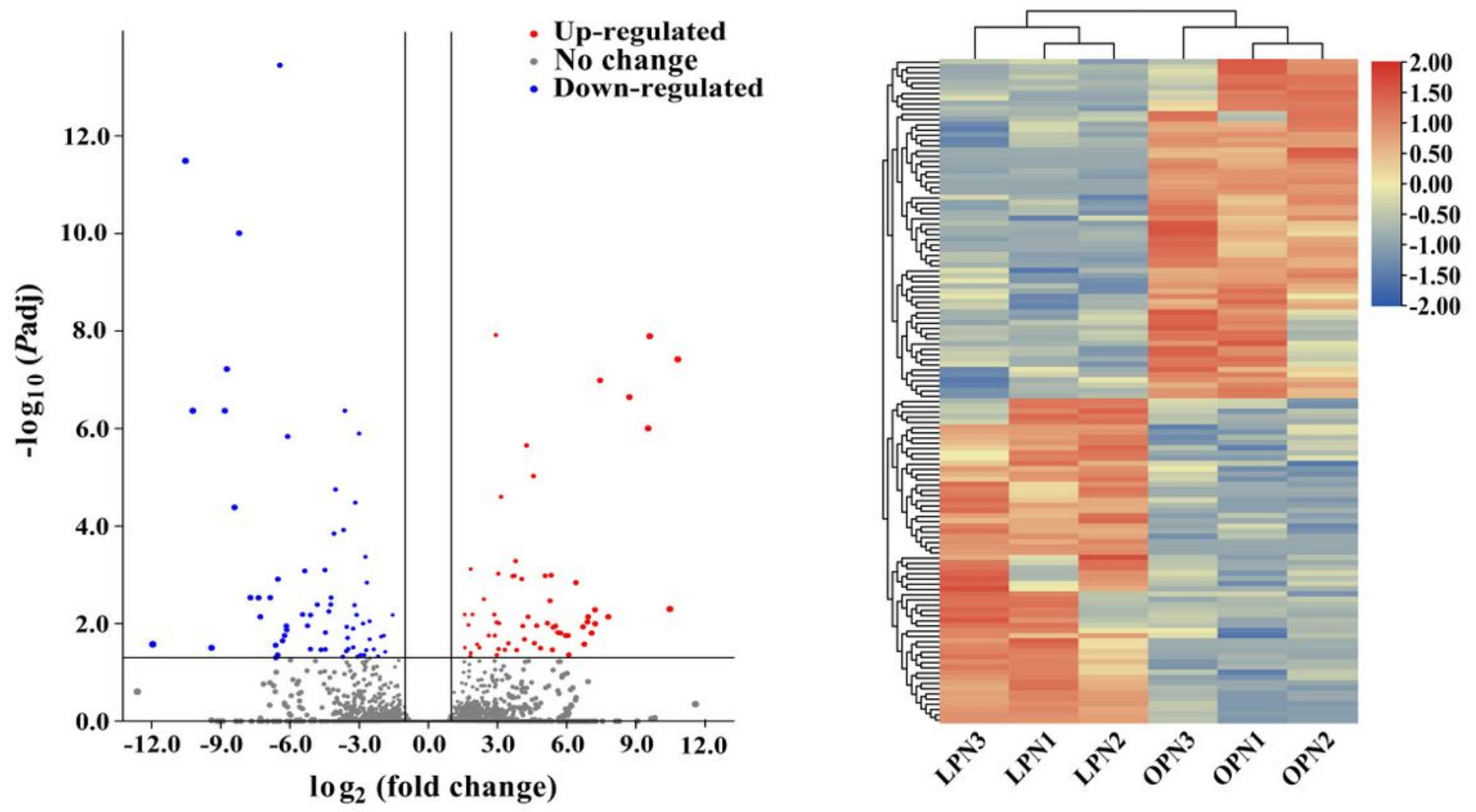

Figure 4

Volcano plot of gene expression levels and heatmap of differentially expressed genes (DEGs). (a) The volcano plot was generated from gene-level differential expression results based on DEseq2, showing the distribution of down- and upregulated genes in LPN as compared with in OPN. DEseq2 showed 65 downregulated and 62 upregulated DEGs in LPN. In blue are the significantly (padj < 0.05 ) downregulated genes with a log2 fold change (LFC) $<-1$ (left of the vertical line). In red are the significantly (padj < 0.05 ) upregulated genes with an LFC > 1 (right of the vertical line). The larger the absolute value of LFC, the larger the area of the point. (b) Clustering heatmap of 127 DEGs. The heatmap shows downregulated and upregulated genes between LPN (LPN1, LPN2 and LPN3) and OPN (OPN1, OPN2 and OPN3) samples (LFC $<-1$ or $>1$, padj $<0.05$ ). Darker blue indicates more significant downregulation, whereas darker red indicates more significant upregulation. 
$\mathbf{a}$

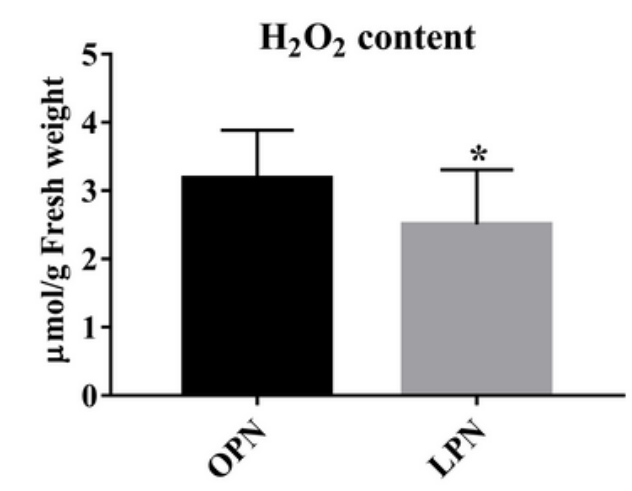

b

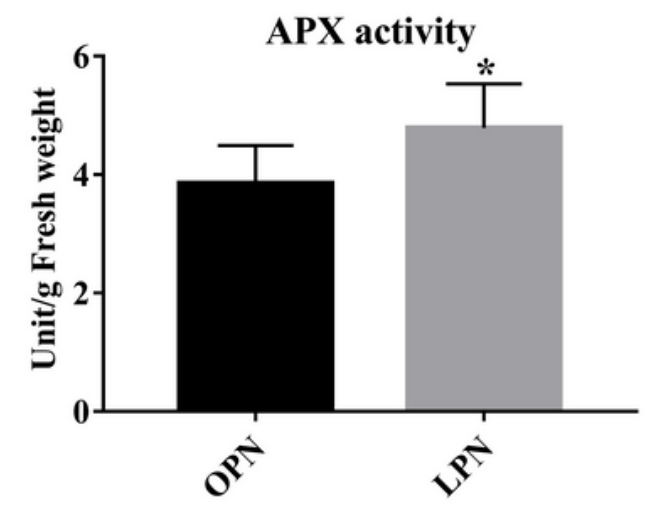

c

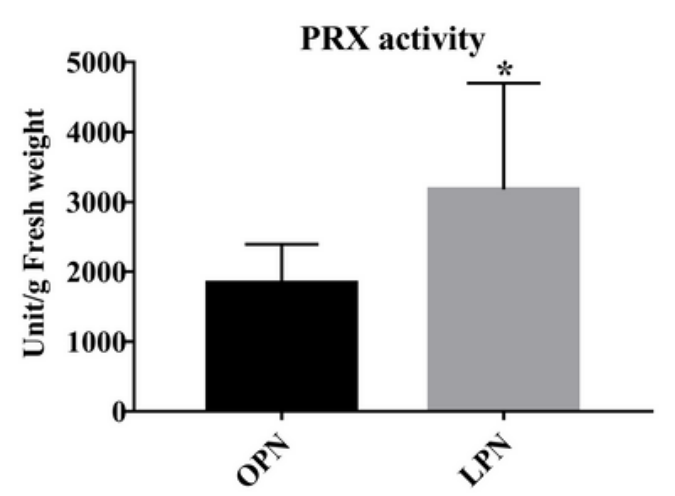

d

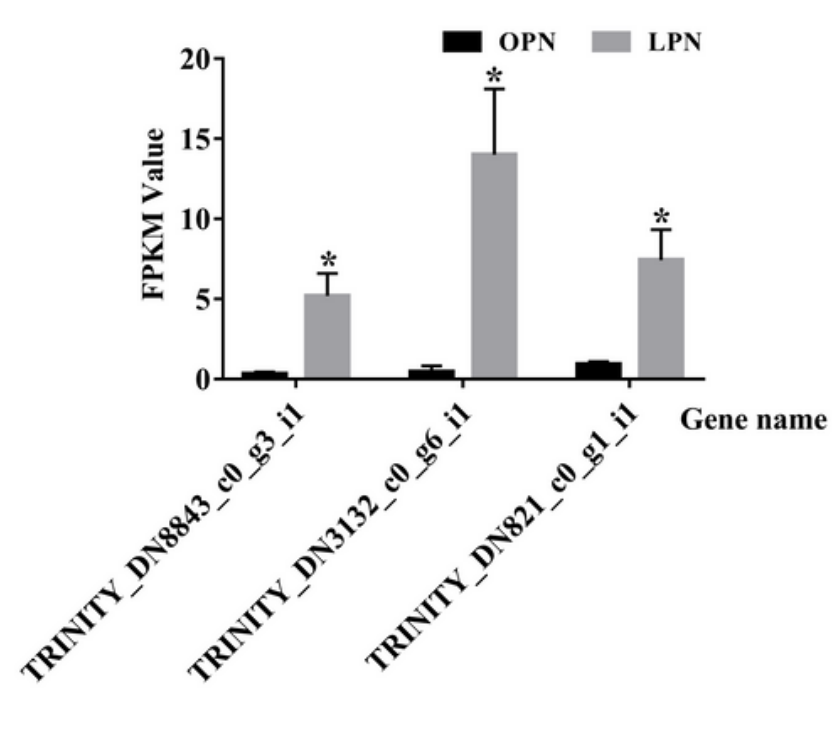

$\mathbf{e}$

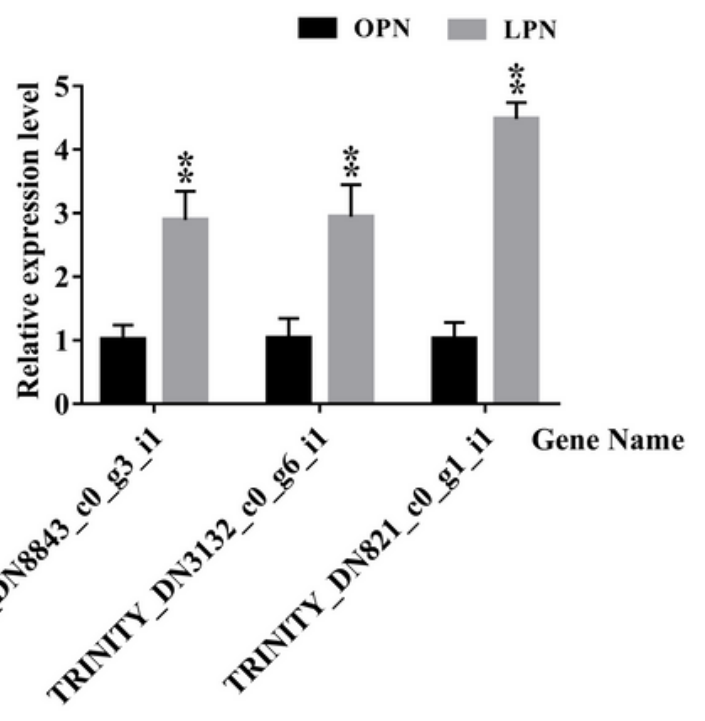

\section{Figure 5}

The determination results of H2O2, APX, PRX, RT-qPCR, and the statistics of FPKM. (a) H2O2 concentration and enzyme activities of (b) APX and (c) PRX, as well as (d) statistics of fragments per kilobase million (FPKM) value of the three genes TRINITY_DN8843_c0_g3_i1 (encoding APX3), TRINITY_DN3132_c0_g6_i1 (encoding PRX45), and TRINITY_DN821_c0_g1_i1 (encoding MPK3) and (e) RT-qPCR validation results. Data are presented as mean \pm s.d. $(n=3)$. The asterisk and double asterisks 
represent significant differences determined by the independent-sample t-test in PASW at $p<0.05\left(^{*}\right)$ and $p<0.01(* *)$, respectively.

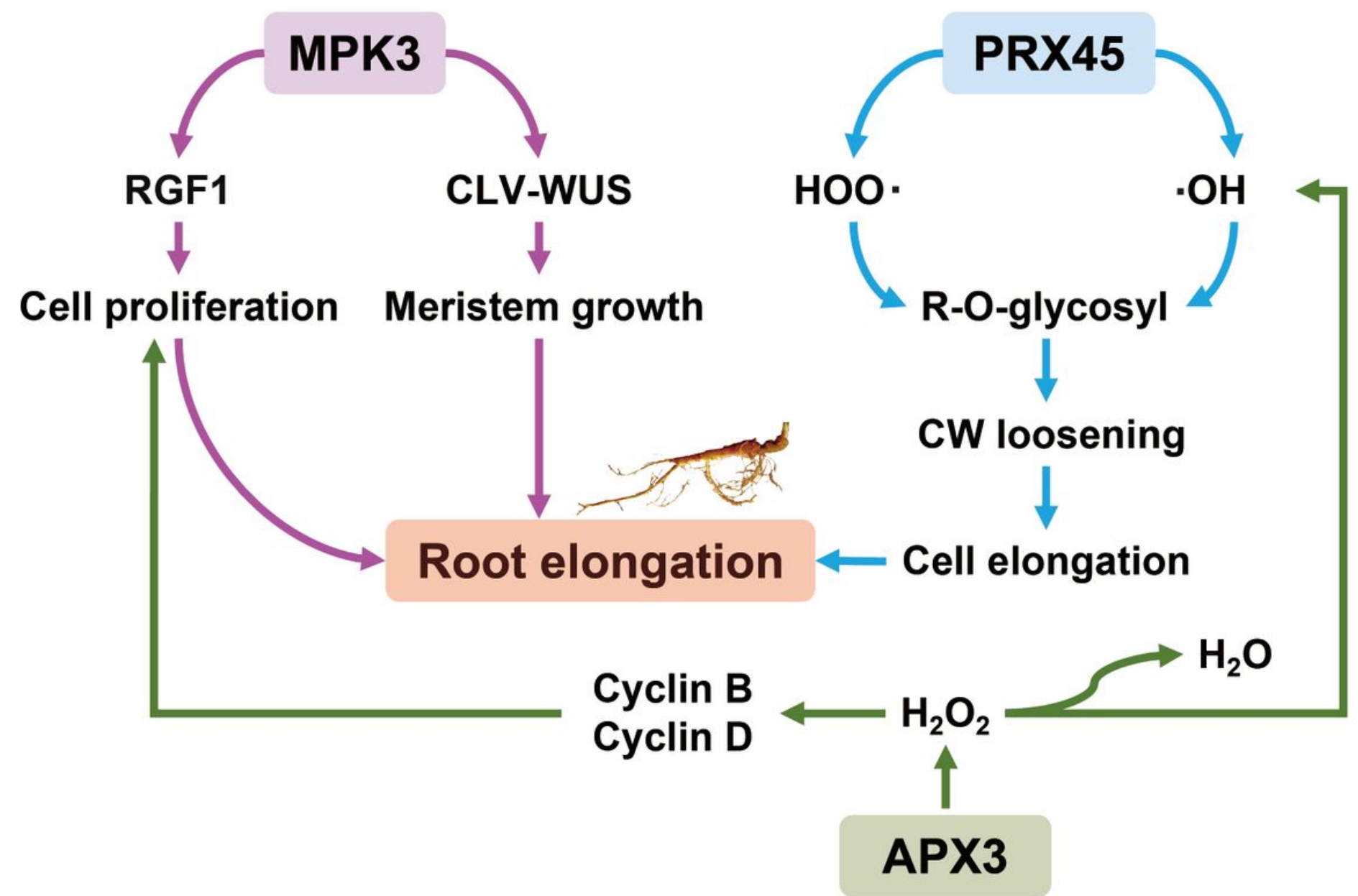

Figure 6

A Putative network diagram of molecular regulatory mechanism leading to LPN formation. The blue, green, and purple arrows represent the metabolic pathways involving PRX45, APX3 and MPK3, respectively.

\section{Supplementary Files}

This is a list of supplementary files associated with this preprint. Click to download.

- SupplementaryFigure1.png

- SupplementaryTable1.docx

- SupplementaryTable2.docx 